Normalisierung phantasmatischer Geschlechtskörper? Oder: warum Schönheitschirurgie (nicht) als symbolische Gewalt gefasst wird. Rezension zu: Villa, P. (Hg.): Schön normal. Manipulation am Körper als Technologie des Selbst

Duttweiler, S

DOI: https://doi.org/10.1007/s11614-011-0010-6

Posted at the Zurich Open Repository and Archive, University of Zurich ZORA URL: https://doi.org/10.5167/uzh-57512

Journal Article

Published Version

Originally published at:

Duttweiler, S (2011). Normalisierung phantasmatischer Geschlechtskörper? Oder: warum Schönheitschirurgie (nicht) als symbolische Gewalt gefasst wird. Rezension zu: Villa, P. (Hg.): Schön normal. Manipulation am Körper als Technologie des Selbst. Österreichische Zeitschrift für Soziologie (ÖZS) Sonderheft Symbolische Gewalt, 36:117-119.

DOI: https://doi.org/10.1007/s11614-011-0010-6 


\title{
Normalisierung phantasmatischer Geschlechtskörper? Oder: Warum Schönheitschirurgie (nicht) als symbolische Gewalt gefasst wird
}

\author{
Rezension zu: Paula-Irene Villa (Hrsg.) (2008): Schön \\ normal. Manipulation am Körper als Technologie des \\ Selbst. Bielefeld: transcript, 279 Seiten, EUR 28,80
}

\section{Stefanie Duttweiler}

Fitnessbesessene Juristen, Michael Jacksons Nase, THE SWAN und ganz normale Frauen, die sich unters Messer legen, ethnisierte und phantasmatische Geschlechtskörper, Foucault, Butler und immer wieder das unternehmerische Selbst - von symbolischer Gewalt ist in diesem Buch an keiner Stelle die Rede. Und dennoch lässt sich der Tenor des gesamten Sammelbandes „Schön normal. Manipulation am Körper als Technologie des Selbst“ als Problematisierung symbolischer Gewalt lesen, die „schön normale Körper“ als unhintergehbare Norm setzt und in vielfältigen Technologien des Körpers und des Selbst wirksam wird.

Die instruktive Einleitung von Paula-Irene Villa fasst schon im Titel „Wider die Rede vom Äußerlichen“ das Thema des Bandes präzise zusammen: Alle Fallstudien kreisen um das Thema der Verkörperung sozialer Normen und damit auch um den Kern symbolischer Gewalt - die Komplizenschaft zwischen Herrschenden und Beherrschten: Menschen unterwerfen sich freiwillig herrschenden Schönheits-, Geschlechts- und Rassennormen. Wie ist diese (vermeintlich?) selbstbestimmte Entscheidung zu (operativen) Körpermanipulationen analytisch zu fassen?

Der Gegenstand des für alle an Körper- und Geschlechtersoziologie interessierten, lesenswerten Buches ist klug gewählt: Schönheitshandeln und Schönheitschirurgie erweisen sich als provozierende Phänomene, an denen sich die Ambivalenz von individueller Autonomie und (Selbst-)Unterwerfung des Individuums paradigmatisch zeigt. Die meisten AutorInnen thematisieren explizit diese Gleichzeitigkeit: Als Effekt der Normalisierung (Meili, Morgan, Degele), als Subjektivierung und Begleitphänomen reflexi-

(C) VS Verlag für Sozialwissenschaften 2011

Dr. S. Duttweiler $(\bowtie)$

Institut für Erziehungswissenschaften, Universität Zürich,

Freiestraße 36, 8032 Zürich, Schweiz 
ver Moderne (Villa) oder unter gouvernementalitätstheoretischer Perspektive (Maasen, Fleig, Ullrich). Auch wenn die AutorInnen ihre Ausführungen theoretisch unterschiedlich akzentuieren, ergeben sich dabei doch einige ermüdende Redundanzen - auch mit anderen Studien, die sich auf Technologien des Körpers und des Selbst beziehen.

Interessant wird es m. E. vor allem dort, wo die Beiträge das Spezifische der Schönheitschirurgie hervorheben: Die Herstellung normaler Körper als Körper, denen die Verkörperung sozialer Positionen sowie einer spezifischen geschlechtlichen, rassischen, unternehmerischen, willensstarken Identität zugemutet wird. Die Effekte der diskursiven Kopplung von Selbst und Körper als diskriminierende Zuschreibung beschreibt Kathy Davis in einem kurzen Abriss über die Geschichte der ethnisch kosmetischen Chirurgie. Sie erlaubt marginalisierten Gruppen, deren Körperteile rassisch markiert werden, ein Passing, d. h. eine neue Identität anzunehmen und damit der Unterordnung und Unterdrückung zu entkommen. Dabei werden sie nicht selten als „Verräter ihrer Rasse“ diffamiert. Insbesondere bei Michael Jackson ist dies augenfällig, obwohl sich seine diversen Schönheitsoperationen in vielerlei Hinsicht beobachten lassen (Verwischung der Kategorien Hetero-Homosexualität, Mann-Frau, Schwarz-Weiss, Publicitystrategie, selbstbestimmtes Experimentieren mit dem eigenen Körper). Davis kommt zu dem Schluss, dass diese Operationen mit der (schmerzlichen) Einsicht konfrontieren, dass in der Gesellschaft Menschen immer noch als Andere definiert und diskriminiert werden - und somit auch gezwungen sind, Wege zu finden, diese Andersheit zu verstecken. Die ,Komplizenschaft der Einzelnen wird somit nicht als Einwilligung verstanden, sondern als Befreiung aus rassistischen Zuschreibungen.

Das Leiden am eigenen Körper, das zeigen auch die meisten anderen Beiträge, macht die Selbstnormalisierung durch Körpermanipulation plausibel. Auch ein Teil der Schönheitschirurgen, die Barbara Meili interviewt hat, bedient sich dieser Legitimationsstrategien (andere fassen Schönheitschirurgie als Dienstleistung mündiger Bürger).

Auch der fitness- und körperbesessene Protagonist Philipp in John von Düffels Roman EGO (Anne Fleig) leidet; überpointiert und parodistisch wird gezeigt, wie hilflos die Verdrängung des Leidens an der Unverfügbarkeit des Lebens und des eigenen Körpers durch Körperbearbeitung letztlich ist. Glücklicher (?) endet die (v)erdichtete Geschichte der fettleibigen Josephine. Morgan kann an deren kompetenten Umgang mit ihrem Leiden minutiös zeigen, wie der systematische Fett-Hass im gegenwärtigen Amerika Personen wie Josephine dazu veranlasst, eine Schlankheits-Magenoperation selbst zu wollen. Die Entscheidung für eine Operation gleicht einem Weg vom Sünder zum weltlichen Heiligen und verspricht eine körperlich, erotisch und sozial bessere Zukunft. Dabei operiert der Diskurs mit vertrauten Elementen: Transzendenz, Befreiung und Selbstermächtigung. Aufschlussreich ist auch ihre Übertragung des vierphasigen Entwicklungsschemas, das Elisabeth Beck-Gernsheim für Reproduktionsmedizin erarbeitet hat: von der Erfindung neuer technologischer Verfahren, Neukategorisierungen und Diskursverschiebungen, Normalisierung und der Schaffung eines Marktes hin zu einer forcierten Anwendung, Regulierung und Überwachung. Zwar sei Phase vier noch in weiter Ferne, aber, so Davis, sie nähert sich.

Dass das Leiden nicht nur die Entscheidung einer Operation, sondern auch Kern der operativen Materialisierung von Geschlecht darstellt, zeigt der ausgezeichnete Artikel des Literaturwissenschaftlers Simon Strick. In seiner Analyse der Dramaturgie der Rea- 
lity-Show „THE SWAN - endlich schön!“ zeigt er, wie das kosmetisch hergestellte neue weiblichere Selbst durch „Leidensarbeit“ und die symbolische Verknüpfung von Schmerz und Weiblichkeit eingeleibt und authentifiziert wird. Das Setting der Show mit ihrem Kernstück der Operation liest er als Inszenierung eines biographischen Bruchs. Diesen fasst er überzeugend als ein tatsächliches Zerbrechen. Im Akt der Operation kommt es zu einer schrittweisen Reduktion der Kandidatinnen auf den bloßen Körper und damit einer „Auslöschung des Körperselbst“. Aufbauend auf diesem Punkt der Ohnmacht und des Nicht-Seins kommt es zu einer doppelten Resignifizierung: im chirurgischen Akt selbst, der eine kohärente, sexualisierte Weiblichkeit herstellt, sowie durch die Schaffung inszenatorischer Situationen heterosexueller, emanzipativ-weiblicher oder familiär-mütterlicher Weiblichkeit.

In einer Mischung aus Melodrama, Horror- und Pornofilm wird die Operation als Narrationsamalgam aus ,sex, violence and emotion“ inszeniert und verleitet zu einer Identifikation auf der Körperebene: Wir sind schockiert von den brutalen Bildern und leiden mit. Strick spricht von viszeraler Identifikation, die notwendig ist, um der entkörperlichten Fleischlichkeit auf dem OP-Tisch Körperlichkeit und Menschlichkeit zuzusprechen. Diese Anerkennung ist es, die die (Neu-)Aneignung des weiblichen Körperselbst authentifiziert.

Die Aneignung des neuen Geschlechtsselbst verläuft also über das inszenatorische Arrangement von Leiden, Ohnmacht und Auslöschung als spezifische Bedingung der weiblichen Verkörperung. Damit erweist sie sich so als ,präzise Gegenposition“ (Strick) zu Positionen, die Freiheit und Selbstbestimmung präferieren sowie zum Argument von Kathy Davis (dem sich auch Sabine Maasen in ihrem Beitrag anschließt), im Leiden am Diskurs ein Moment der Selbstbestimmung zu sehen.

Insgesamt wird nach der Lektüre des Buches die Frage drängend, warum Schönheitschirurgie an keiner Stelle (explizit) als symbolische Gewalt interpretiert wird. Legt der Begriff eine Passivität und ,Opferhaltung' nahe, die zu unterstellen heute weder opportun noch theoretisch befriedigend ist? Offenbar kann in der Theoriearbeit auf die Figur der Ambivalenz, die Selbstbestimmung und -ermächtigung stark macht, nicht verzichtet werden. Doch wie die provozierenden Arbeiten der Künstlerin Valie Export (thematisiert von Markus Brunner) zeigen, kann die Manipulation des eigenen Körpers die symbolische Gewalt auch aufdecken und in Frage stellen: Ihre Arbeiten machen sie sicht- und fühlbar, indem sie leibliche Reaktionen provozieren, die einen Erkenntnisprozess in Gang setzen. Exports künstlerisches Werk thematisiert somit auch das Leiden an der Unmöglichkeit eines ,richtigen Lebens im falschen' - und erschöpft sich nicht im Aufzeigen der Ambivalenz von Unterdrückung und Selbstbestimmung. Ob auch mit dem Begriff der symbolischen Gewalt darüber hinaus zu gelangen wäre? 